\title{
Gender Modulates the APOE $\varepsilon 4$ Effect in Healthy Older Adults: Convergent Evidence from Functional Brain Connectivity and Spinal Fluid Tau Levels
}

\author{
Jessica S. Damoiseaux, ${ }^{1}$ William W. Seeley, ${ }^{2}$ Juan Zhou, ${ }^{3}$ William R. Shirer, ${ }^{1}$ Giovanni Coppola, ${ }^{4}$ Anna Karydas, ${ }^{2}$ \\ Howard J. Rosen, ${ }^{2}$ Bruce L. Miller, ${ }^{2}$ Joel H. Kramer, ${ }^{2}$ and Michael D. Greicius, ${ }^{1}$ for the Alzheimer's Disease \\ Neuroimaging Initiative \\ ${ }^{1}$ Functional Imaging in Neuropsychiatric Disorders Laboratory, Department of Neurology and Neurological Sciences, Stanford University School of \\ Medicine, Stanford, California 94305, ${ }^{2}$ Memory and Aging Center, Department of Neurology, University of California, San Francisco, California 94143-1207, \\ ${ }^{3}$ Neuroscience and Behavioral Disorders Program, Duke-National University of Singapore Graduate Medical School, Singapore 169857, and ${ }^{4}$ Semel \\ Institute for Neuroscience and Human Behavior, Departments of Psychiatry and Neurology, David Geffen School of Medicine, University of California, Los \\ Angeles, California 90095
}

We examined whether the effect of the apolipoprotein E (APOE) genotype on functional brain connectivity is modulated by gender in healthy older human adults. Our results confirm significantly decreased connectivity in the default mode network in healthy older $A P O E$ $\boldsymbol{\varepsilon} 4$ carriers compared with $\varepsilon 3$ homozygotes. More important, further testing revealed a significant interaction between $A P O E$ genotype and gender in the precuneus, a major default mode hub. Female $\varepsilon 4$ carriers showed significantly reduced default mode connectivity compared with either female $\boldsymbol{\varepsilon} 3$ homozygotes or male $\boldsymbol{\varepsilon} 4$ carriers, whereas male $\boldsymbol{\varepsilon} 4$ carriers differed minimally from male $\boldsymbol{\varepsilon} 3$ homozygotes. An additional analysis in an independent sample of healthy elderly using an independent marker of Alzheimer's disease, i.e., spinal fluid levels of tau, provided corresponding evidence for this gender-by- $A P O E$ interaction. Together, these results converge with previous work showing a higher prevalence of the $\boldsymbol{\varepsilon} 4$ allele among women with Alzheimer's disease and, critically, demonstrate that this interaction between $A P O E$ genotype and gender is detectable in the preclinical period.

\section{Introduction}

The most important known risk factor for Alzheimer's disease (AD) is the presence of the apolipoprotein E type 4 allele (APOE $\varepsilon 4)$ (Strittmatter et al., 1993). Carriers of the $\varepsilon 4$ allele have an

Received Jan. 20, 2012; revised April 17, 2012; accepted April 18, 2012.

Author contributions: W.W.S., B.L.M., J.H.K., and M.D.G. designed research; W.W.S., J.Z., G.C., A.K., and J.H.K. performed research; J.Z., W.R.S., G.C., A.K., H.J.R., and B.L.M. contributed unpublished reagents/analytic tools; I.S.D. analyzed data; J.S.D. and M.D.G. wrote the paper.

This work was supported by the Hillblom Foundation, the John Douglas French Alzheimer's Disease Foundation, the JNA Foundation, and National Institutes of Health Grants NS073498 and AG032306. We thank William Irwin, Maria Molfino, and Heidi Jiang for their help with MRI data transfer and preprocessing; and Eric Klein for his help with the APOE genotyping. Data collection and sharing for this project was funded by the Alzheimer's Disease Neuroimaging Initiative (ADNl; National Institutes of Health Grant U01 AG024904). The Canadian Institutes of Health Research provides funds to support ADNI clinical sites in Canada. Private sector contributions are facilitated by the Foundation for the National Institutes of Health. The grantee organization is the Northern California Institute for Research and Education, and the study is coordinated by the Alzheimer's Disease Cooperative Study at the University of California, San Diego. ADNI data are disseminated by the Laboratory for Neuroimaging at the University of California, Los Angeles. Cerebrospinal data used in the preparation of this article were obtained from the Alzheimer's Disease Neuroimaging Initiative (ADNI) database (www.loni.ucla.edu/ADNI). As such, ADNI investigators contributed to the design and implementation of ADNI and/or provided data but did not participate in the analysis or writing of this report. A complete listing of ADNI investigators can be found at http://adni.loni.ucla.edu/wpcontent/uploads/how_to_apply/ADNI_Acknowledgement_List.pdf.

The authors declare no competing financial interests.

Correspondence should be addressed to either Jessica S. Damoiseaux or Michael D. Greicius, Functional Imaging in Neuropsychiatric Disorders Laboratory, Department of Neurology and Neurological Sciences, Stanford University School of Medicine, 780 Welch Road, Palo Alto, CA 94304. E-mail: jeske@stanford.edu or greicius@stanford.edu. DOI:10.1523/JNEUROSCI.0305-12.2012

Copyright $\odot 2012$ the authors $\quad 0270-6474 / 12 / 308254-09 \$ 15.00 / 0$ increased risk of developing for AD compared to with people who are homozygous for the $\varepsilon 3$ allele (the most common APOE genotype) (Farrer et al., 1997). An important observation that tends to be overlooked is that this $A P O E$ effect varies with gender. Women are more likely to develop AD than men across most ages and APOE genotypes (Farrer et al., 1997), but this difference is significantly more pronounced in persons with the APOE $\varepsilon 3 / \varepsilon 4$ genotype; women with the $A P O E \varepsilon 3 / \varepsilon 4$ genotype are at approximately fourfold increased risk, but men with it show little to no increased risk (Payami et al., 1996; Farrer et al., 1997; Bretsky et al., 1999). In addition to having an increased risk for AD, female $\varepsilon 4$ carriers with mild cognitive impairment (MCI), a precursor to $\mathrm{AD}$, also express more prominent phenotypic features than their male counterparts, such as lower hippocampal volumes and worse cognitive scores (Fleisher et al., 2005). A large autopsy series found that female $\varepsilon 4$ carriers also had the greatest amyloid plaque and neurofibrillary tangle pathology (Corder et al., 2004). To date, this interaction between gender and APOE status, evident in AD risk, MCI phenotypes, and postmortem data, has not been explored in vivo in healthy older subjects.

Previous resting state functional magnetic resonance imaging (fMRI) studies have shown that functional connectivity changes in the default mode network (DMN) in MCI (Sorg et al., 2007) and $\mathrm{AD}$ (Greicius et al., 2004; Wang et al., 2007; Zhang et al., 2009) and that this default mode functional connectivity deteriorates as the disease progresses (Bai et al., 2011; Damoiseaux et 
Table 1. Demographics and neuropsychological measures

\begin{tabular}{|c|c|c|c|c|}
\hline & $\begin{array}{l}\epsilon 3 \\
\text { Homozygotes }\end{array}$ & $\epsilon 4$ Carriers & $\begin{array}{l}\epsilon 3 \text { Homozygotes } \\
\text { vs } \epsilon 4 \text { carriers ( } p \text { ) }\end{array}$ & $\begin{array}{l}A P O E \times \text { gender } \\
\text { interaction }(p)\end{array}$ \\
\hline \multicolumn{5}{|l|}{ Imaging dataset } \\
\hline Gender (m/f) & $34 / 54$ & $26 / 17$ & $0.019^{*}\left(\right.$ Pearson $\left.\chi^{2}\right)$ & - \\
\hline Age (years) & $70.8 \pm 6.9$ & $70.2 \pm 6.9$ & 0.659 & 0.413 \\
\hline Education (years)* & $17.5 \pm 2.1$ & $17.0 \pm 2.6$ & 0.240 & 0.917 \\
\hline MMSE & $29.3 \pm 0.9$ & $29.2 \pm 1.0$ & 0.490 & 0.754 \\
\hline CVLT immediate recall & $11.1 \pm 2.8$ & $10.8 \pm 3.5$ & 0.879 & 0.329 \\
\hline CVLT delayed recall & $11.7 \pm 3.1$ & $11.8 \pm 3.3$ & 0.622 & 0.315 \\
\hline $\begin{array}{l}\text { Benson Complex Figure } \\
\text { recall }\end{array}$ & $11.4 \pm 2.5$ & $11.3 \pm 2.4$ & 0.934 & 0.639 \\
\hline \multicolumn{5}{|l|}{ ADNI dataset } \\
\hline Gender (m/f) & $32 / 35$ & $17 / 9$ & $0.127\left(\right.$ Pearson $\left.\chi^{2}\right)$ & - \\
\hline Age (years) & $75.9 \pm 5.0$ & $75.9 \pm 5.8$ & 0.919 & 0.723 \\
\hline Education (years)* & $16.1 \pm 2.5$ & $15.6 \pm 3.1$ & 0.269 & 0.358 \\
\hline MMSE & $29.2 \pm 1.0$ & $29.1 \pm 0.8$ & 0.815 & 0.432 \\
\hline
\end{tabular}

CVLT, California Verbal Learning Test; MMSE, Mini Mental State Examination; $m$, male; f, female. *Significant at $p<0.05$.

al., 2011). Recent resting state fMRI studies have investigated whether we can detect similar changes in brain functional connectivity in healthy older $\varepsilon 4$ carriers (Sheline et al., 2010; Machulda et al., 2011; Trachtenberg et al., 2011; Westlye et al., 2011). Trachtenberg et al. (2011) found no APOE-related differences in DMN connectivity. The other three studies show significant DMN connectivity differences between $\varepsilon 4$ carriers and $\varepsilon 3$ homozygotes, although there is substantial variability in their results. None of these studies examined whether the observed default mode functional connectivity differences varied by gender.

In this study, we examined the effect of $A P O E$ genotype and gender on default mode functional connectivity in healthy older subjects. In line with previous research, we expected to see a pattern similar to what we have observed in $\mathrm{AD}$; that is, decreased connectivity in the DMN (Greicius et al., 2004; Damoiseaux et al., 2011), with the greatest effect in female $\varepsilon 4$ carriers. To obtain convergent evidence for these imaging findings, we also tested the same model in an analysis of spinal fluid protein levels in healthy older controls from the Alzheimer's Disease Neuroimaging Initiative (ADNI) dataset.

\section{Materials and Methods}

Participants in the University of California, San

Francisco/Stanford study

Healthy older adults were recruited as part of longitudinal study of normal aging at the University of California, San Francisco (UCSF). All participants provided informed consent according to the Declaration of Helsinki, and the Institutional Review Board at UCSF approved the procedures. Exclusion criteria for this study were the following: left-handedness; any significant medical, neurological, or psychiatric illness; history of brain damage; use of psychoactive medication; poor data quality; and carrying an $\varepsilon 2$ allele [APOE $\varepsilon 2$ may have a protective effect against AD (Scott et al., 1997); therefore, we excluded carriers of this allele from our analyses]. Resting state fMRI data and APOE genotype were available for 234 participants. Of these 234 participants, 27 were excluded because of left-handedness, 22 because of poor data quality (excessive head motion, significant signal loss, partial brain coverage), 36 because of use of psychoactive medication, and 18 because they carried the $\varepsilon 2$ allele. This left us with a total of 131 participants, all of whom were included. Of these 131 participants, 43 were $\varepsilon 4$ carriers. Of those 43,4 were $\varepsilon 4$ homozygotes ( 2 men, 2 women), 39 were $\varepsilon 3 / \varepsilon 4$ homozygotes ( 24 men, 15 women), and 88 were $\varepsilon 3$ homozygotes ( 34 men, 54 women). Table 1 includes the demographic details.

\section{Neuropsychological assessment}

All participants underwent extensive neuropsychological assessment. For this study, we only considered the results of the Mini-Mental State Examination and the neuropsychological tests targeting memory function: the California Verbal Learning Test, both immediate and delayed recall; and the recall of the Benson Complex Figure. Raw test scores were tested for differences between $A P O E$ genotype, gender, and the interaction between the two using a multivariate ANOVA, with $p<0.05$.

\section{APOE genotype assessment}

$A P O E$ single nucleotide polymorphism (SNP) genotyping was performed by real-time PCR on an Applied Biosystems 7900HT RealTime PCR machine using the TaqMan SNP Genotyping Assay (Applied Biosystems) for rs429358 and rs7412 with identification numbers C_3084793_20 and C_904973_10, respectively. The protocol was followed as outlined in the manufacturer's instructions, and every assay was performed in duplicate. In addition to a standard curve amplification protocol, an allelic discrimination step was added to facilitate the contrast between the two alleles and their respective reporter dyes. Sequence Detection Systems Software version 2.3 (Applied Biosystems) was used to analyze the SNP genotyping data.

\section{MRI data acquisition}

fMRI was performed at the UCSF Neuroscience Imaging Center on a 3 tesla Siemens Tim Trio scanner using a standard 12-channel head coil. Thirty-six interleaved axial slices ( $3 \mathrm{~mm}$ thick with a gap of $0.6 \mathrm{~mm}$ ) were imaged parallel to the plane connecting the anterior and the posterior commissures using a $\mathrm{T} 2^{*}$-weighted echoplanar sequence [repetition time (TR), $2000 \mathrm{~ms}$; echo time (TE), $27 \mathrm{~ms}$; flip angle (FA), 80; voxel size, $2.5 \times 2.5 \times 3.6 \mathrm{~mm}$ ]. The field of view was $230 \times 230 \mathrm{~mm}$, and the matrix size was $92 \times 92$. All subjects underwent 8 min resting state fMRI after being instructed only to remain awake with their eyes closed. A volumetric MPRAGE MRI sequence was used to obtain a T1-weighted image of the entire brain in sagittal slices (TR, $2300 \mathrm{~ms}$; TE, $2.98 \mathrm{~ms}$; inversion time, $\left.900 \mathrm{~ms} ; \mathrm{FA}, 9^{\circ}\right)$. The images were reconstructed as a $160 \times 240 \times 256$ matrix with $1 \times 1 \times 1 \mathrm{~mm}$ spatial resolution.

\section{MRI data analysis}

Anatomical data analysis. Anatomical data were analyzed with FMRIB Software Library (FSL)-VBM, a voxel-based morphometry style analysis (Ashburner and Friston, 2000; Good et al., 2001) performed with FSL tools (Smith et al., 2004). First, anatomical images were brain extracted using the Brain Extraction Tool (Smith, 2002). Next, tissue-type segmentation was performed using FAST4 (Zhang et al., 2001). The resultant gray matter partial volume images were then aligned to MNI152 standard space using the affine registration tool FLIRT (Jenkinson and Smith, 2001; Jenkinson et al., 2002), followed by nonlinear registration using FNIRT (Andersson, 2007) (FMRIB technical reports TR07JA1 and TR07JA2; available at www.fmrib.ox.ac.uk/analysis/techrep), which uses a b-spline representation of the registration warp field (Rueckert et al., 1999). For the use of gray matter volume as a voxelwise regressor in the fMRI data analysis, a four-dimensional (4D) image was created by concatenating every participant's standard space gray matter image. For direct comparison of gray matter volume, the individual standard space gray matter images were averaged to create a study-specific template, to which the native gray matter images were then nonlinearly reregistered. The registered partial volume images were then modulated (to correct for local expansion or contraction) by dividing by the Jacobian of the warp field. The modulated segmented images were smoothed with an isotropic Gaussian kernel with $\sigma=3 \mathrm{~mm}$. Finally, to test for significant differences between $\varepsilon 4$ carriers and $\varepsilon 3$ homozygotes, a voxelwise general linear model was applied using permutation-based nonparametric testing, with Threshold-Free Cluster Enhancement (TFCE) as implemented in FSL (Smith and Nichols, 2009) and $p<0.05$ familywise error corrected.

Resting state $f M R I$ data analysis. Image preprocessing was performed using tools from the FSL, version 4.1 (Smith et al., 2004). The following prestatistics processing was applied: motion correction (Jenkinson et al., 2002); removal of nonbrain structures (Smith, 2002); spatial smoothing using a Gaussian kernel of $6 \mathrm{~mm}$ full-width at half-maximum; meanbased intensity normalization of all volumes by the same factor (i.e., 4D 
grand-mean scaling to ensure comparability between datasets at the group level); high-pass temporal filtering (Gaussian-weighted leastsquares straight line fitting, with $\sigma=75.0 \mathrm{~s}$ ); and Gaussian low-pass temporal filtering ( $\sigma=$ $2.8 \mathrm{~s}$ ). After preprocessing, the functional scan was first aligned to the participant's highresolution T1-weighted image, which was subsequently registered to the MNI152 standard space [average T1 brain image constructed from 152 normal subjects at Montreal Neurological Institute (MNI)] using affine linear registration (Jenkinson et al., 2002). To perform voxelwise between-group comparisons of resting state connectivity, the dual regression technique previously described (Filippini et al., 2009; Veer et al., 2010; Damoiseaux et al., 2011) was used. This approach entails three steps. First, create data-driven, populationspecific spatial maps showing large-scale connectivity patterns by running group independent component analysis (ICA) on the concatenated resting state data of equal numbers of $\varepsilon 4$ carriers and $\varepsilon 3$ homozygotes, men, and women (17 subjects per group; 68 total). Equal numbers of subjects per subgroup were used here to avoid introducing bias in the creation of the connectivity networks of interest. The dataset was decomposed into 30 independent components. Second, perform the actual dual regression by using all 30 independent components in a linear model fit (spatial regression) against all 131 individual datasets, resulting in specific time courses for each independent component and subject, and using these time courses in a linear model fit (temporal regression) against the subject's resting state data to estimate subject-specific spatial maps. Third, perform voxelwise between-group statistical testing on the subject-specific spatial maps using nonparametric permutation testing (5000 permutations) (Nichols and Holmes, 2002). To control for differences in gray matter volume, the individual gray matter partial volume maps were included as a voxelwise regressor in the betweengroup comparison, as described by Oakes et al. (2007) and implemented in FSL.

The DMN and a control network in which we did not expect to find any changes (i.e., the primary visual network) were selected for betweengroup analyses. In line with previous observations (Damoiseaux et al., 2011; Westlye et al., 2011), the DMN was divided into three subnetworks (referred to here as the anterior, posterior, and ventral DMNs), identified by visual inspection of the group ICA. To assess any potential differences in modulation of these networks, we included all three networks in the analysis plus the primary visual network (identified by visual inspection as a single independent component). Figure 1 depicts a visual representation of all the networks included in the analyses. Fractionation of the DMN into several subnetworks has been observed in multiple previous studies that used a different methodology (i.e., seed-based correlations), studied a different population (i.e., younger healthy adults), or did both (Uddin et al., 2009; Andrews-Hanna et al., 2010; Littow et al., 2010; Westlye et al., 2011). In addition, subdivisions of the default mode system have been associated with distinct cognitive functions (Uddin et al., 2009; Andrews-Hanna et al., 2010; Qin et al., 2011; Whitfield-Gabrieli et al., 2011). We therefore believe that this fractionation is not merely a methodological artifact, but that it represents resting state networks with distinct functional attributes.

To find significant differences between $\varepsilon 4$ carriers and $\varepsilon 3$ homozygotes, a voxelwise general linear model was applied using permutation-based, non-

B
22 $-4$

$-22$
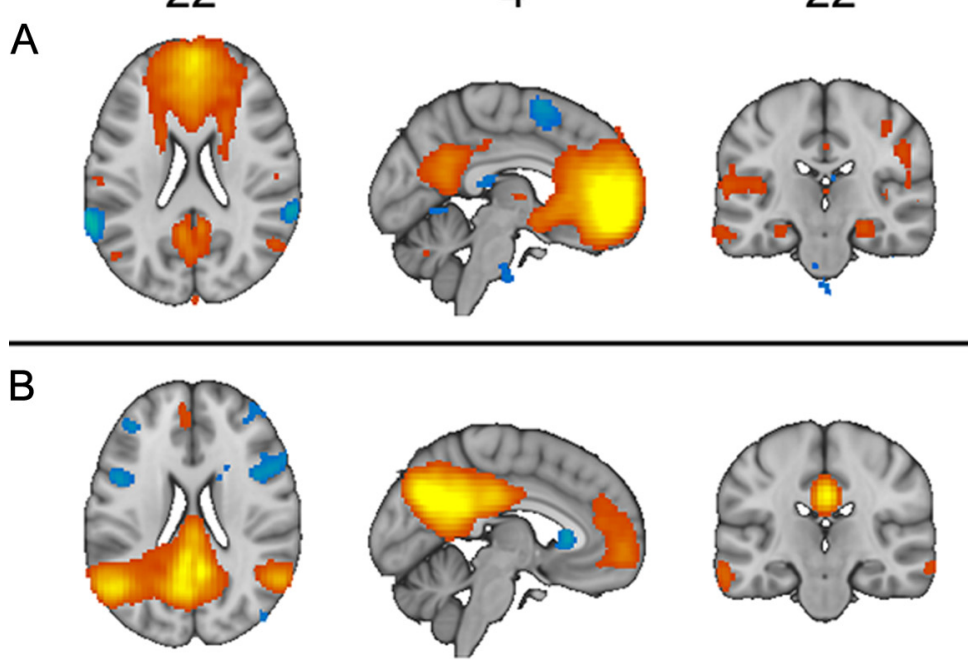

(1)
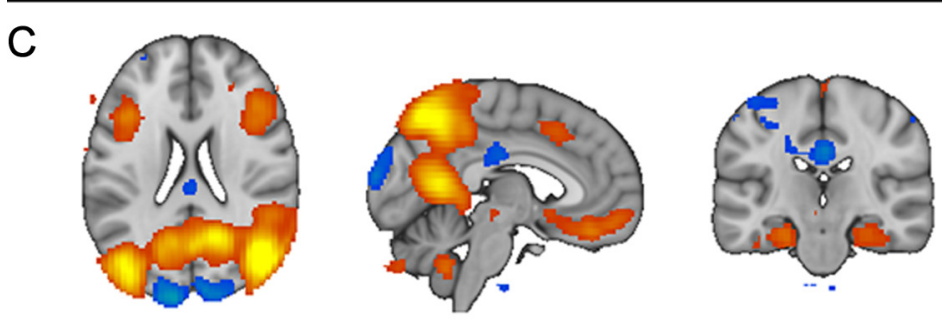

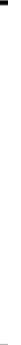
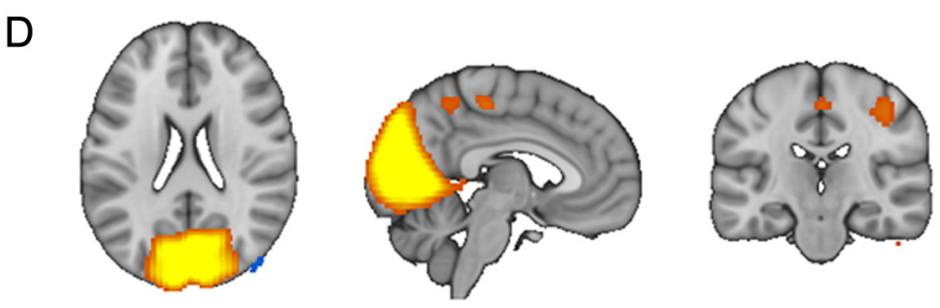

Figure 1. Spatial maps of the networks of interest and the control network. $\boldsymbol{A}-\boldsymbol{D}$, The group independent components selected primary visual network as a control (D). All are shown here using a posterior probability threshold of $p>0.5$; MNI coordinates (in millimeters; numbers at the top) of the axial, sagittal, and coronal slices. Images are displayed in neurological convention.

parametric testing, using TFCE with $p<0.05$ familywise error corrected, spatially masked with the thresholded group ICA component in question (posterior probability threshold of $p>0.5$ ), and a cluster size $>10$ voxels. Given our a priori hypothesis and the reduced $n$ when splitting by gender, we used TFCE with a more lenient threshold of $p<0.01$ uncorrected, spatially masked with the thresholded group ICA component in question and a cluster size $>10$ voxels for the genotype-by-gender interaction analysis and subsequent post hoc comparisons.

\section{ADNI data}

Data used in this part of the study were obtained from the ADNI database (adni.loni.ucla.edu). The primary goal of ADNI has been to test whether serial MRI, PET, other biological markers, and clinical and neuropsychological assessment can be combined to measure the progression of MCI and early AD. Determination of sensitive and specific markers of very early $\mathrm{AD}$ progression is intended to aid researchers and clinicians to develop new treatments, monitor their effectiveness, and lessen the time and cost of clinical trials.

ADNI is the result of efforts of many coinvestigators from a broad range of academic institutions and private corporations, and subjects have been recruited from $>50$ sites across the United States and Canada. The initial goal of ADNI was to recruit 800 adults, ages 55-90 years, to participate in the research: $\sim 200$ cognitively normal older persons to be 
A

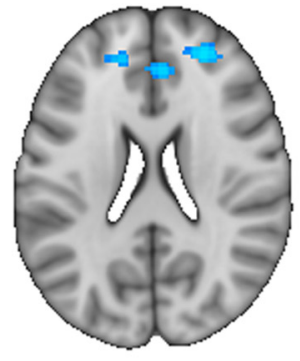

22

B

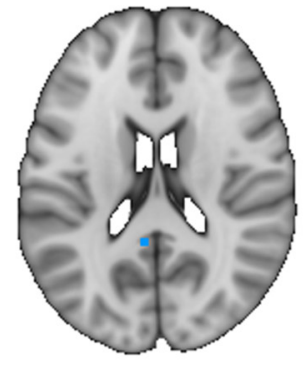

19

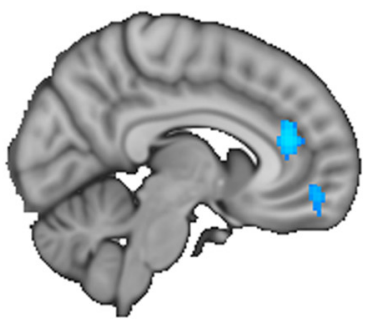

6

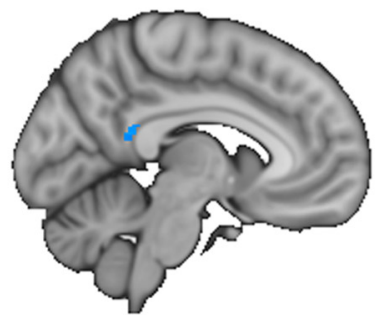

$-6$

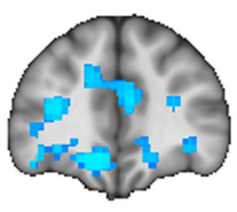

39

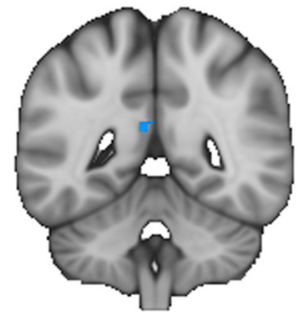

$-46$

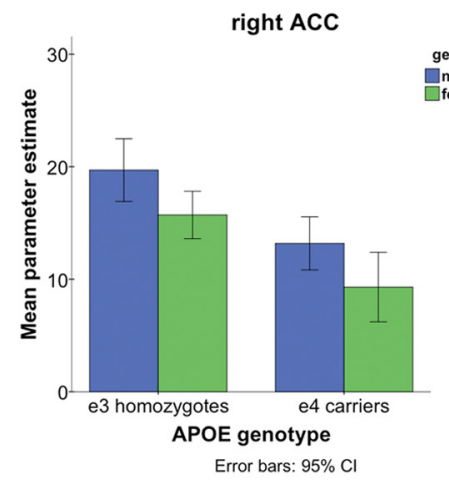

left PCC

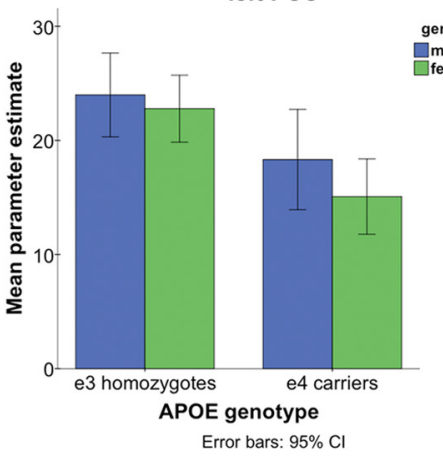

Figure 2. $A P O E \varepsilon 4$ reduces default mode connectivity in healthy older adults. $\boldsymbol{A}, \boldsymbol{B}$, Functional connectivity is decreased in $\varepsilon 4$ carriers compared with $\varepsilon 3$ homozygotes in both the anterior $(\boldsymbol{A})$ and the posterior $(\boldsymbol{B})$ DMN. Bar graphs depict the distribution of the effects across subgroups. They show the mean parameter estimates of a selected region within the anterior DMN (right anterior cingulate gyrus) and the left posterior cingulate cluster within the posterior DMN for male and female $\varepsilon 3$ homozygotes and male and female $\varepsilon 4$ carriers. The difference across both genotype ( $p<$ 0.001 ) and gender $(p=0.005)$ is significant for the anterior cingulate gyrus. For the posterior cingulate gyrus, only the difference across genotype is significant ( $p=0.001)$. The statistical maps, thresholded using TFCE and $p<0.05$ familywise error corrected, are overlaid on the MNI152 brain; MNI coordinates (in millimeters) of the slices are displayed. Images are displayed in neurological convention.

followed up for 3 years, 400 people with MCI to be followed up for 3 years, and 200 people with early AD to be followed up for 2 years. These initial goals were met, and the ADNI project has now been extended as ADNI-2.

Subjects. Within the ADNI database, three separate spreadsheets hold CSF measures; for our analyses, we used the original ADNI baseline data from the "upennbiomark" spreadsheet. In this spreadsheet, 114 healthy controls had CSF data. Of these 114 subjects, 21 were $\varepsilon 2$ carriers and therefore were excluded from our analyses. The remaining subjects were included in our analysis. Of these 93 subjects, 26 were $\varepsilon 4$ carriers ( $2 \varepsilon 4$ homozygotes, 1 man and 1 woman; $24 \varepsilon 3 / \varepsilon 4$ homozygotes, 16 men and 8 women) and 67 were $\varepsilon 3$ homozygotes ( 32 men and 35 women) (Table 1 ).

CSF analysis. CSF samples were collected at different ADNI sites as described in the ADNI procedures manual (http://www.adni-info.org/). Samples were then shipped overnight on dry ice to the ADNI Biomarker Core Laboratory at the University of Pennsylvania Medical Center. All analyses, as described by Shaw et al. (2009), were performed at this location. Mean levels of total tau, amyloid $\beta_{1-42}\left(\mathrm{~A} \beta_{1-42}\right)$, and phospho-tau (p-tau), in picograms per milliliter, were tested for differences between $A P O E$ genotype, gender, and the interaction between the two, using multivariate ANOVA with $p<0.05$. Subsequent post hoc comparisons were performed using the Mann-Whitney $U$ test (one-tailed).

\section{Results}

\section{Demographics}

We did not find a significant difference between $A P O E$ groups in either age or years of education. The distribution of gender across the APOE groups did differ (Pearson $\chi^{2}, p=0.019$ ). No interaction between $A P O E$ genotype and gender was observed for any of these measures (Table 1).

\section{Neuropsychology}

No significant between-group differences or APOE genotype by gender interactions were found for Mini-Mental State Examination scores or memory performance, as measured with the California Verbal Learning Test, immediate and delayed recall, and the recall of the Benson Complex Figure (Table 1).

\section{APOE $\varepsilon 4$ effect on functional connectivity}

We first compared functional connectivity within our four networks among all $\varepsilon 4$ carriers and all $\varepsilon 3$ homozygotes while correcting voxelwise for gray matter volume. We found decreased connectivity in $\varepsilon 4$ carriers compared with $\varepsilon 3$ homozygotes in two large frontal clusters of the anterior DMN (Fig. 2A, Table 2) and in a posterior cingulate cluster of the posterior DMN (Fig. $2 B$, Table 2) using TFCE and $p<0.05$ familywise error correction. No differences were observed in the opposite direction, and no differences were observed in either the ventral DMN or the primary visual network.

To visualize the distribution of the observed effect across genotype and gender, we extracted the mean parameter estimate per group for a significant cluster in each network that showed significant differences between $\varepsilon 4$ carriers and $\varepsilon 3$ homozygotes (note: these are the individual values before the gray matter volume correction is applied). For the anterior DMN, we selected a representative cluster in the right anterior cingulate gyrus, and for the posterior DMN we selected a representative cluster in the left posterior cingulate gyrus. In both clusters, women carrying the $\varepsilon 4$ allele had the lowest mean value (Fig. 2, bar graphs). The 
Table 2. Brain clusters showing significant differences between $A P O E \in 3$ homozygotes and $\epsilon 4$ carriers

\begin{tabular}{|c|c|c|c|c|c|c|}
\hline \multirow[b]{2}{*}{ Brain network } & \multirow[b]{2}{*}{ Contrast } & \multirow[b]{2}{*}{ Brain region } & \multirow[b]{2}{*}{ Cluster size (voxels) } & \multicolumn{3}{|c|}{ Peak MNI coordinates (mm) } \\
\hline & & & & $x$ & $y$ & $z$ \\
\hline Anterior DMN & $\epsilon 3$ Homozygotes $>\epsilon 4$ carriers & $\begin{array}{l}\text { RH frontal pole/anterior cingulate/medial prefrontal } \\
\text { LH frontal pole/medial prefrontal }\end{array}$ & $\begin{array}{r}1435 \\
945\end{array}$ & $\begin{array}{r}26 \\
-30\end{array}$ & $\begin{array}{l}46 \\
44\end{array}$ & $\begin{array}{r}24 \\
-10\end{array}$ \\
\hline Posterior DMN & $\epsilon 3$ Homozygotes $>\epsilon 4$ carriers & LH posterior cingulate gyrus & 17 & -6 & -48 & 18 \\
\hline
\end{tabular}

LH, Left hemisphere; RH, right hemisphere.

difference across both genotype $(p<0.001)$ and gender $(p=$ $0.005)$ was significant for the anterior cingulate gyrus. For the posterior cingulate gyrus, only the difference across genotype was significant $(p=0.001)$.

\section{$A P O E$ genotype and gender interaction}

We then tested voxelwise for an $A P O E$-by-gender interaction in the anterior and posterior DMNs, the two networks that showed an $\varepsilon 4$ effect, again correcting voxelwise for gray matter volume. This analysis revealed a significant interaction in the precuneus, a major hub within the posterior DMN (Hagmann et al., 2008) (Fig. 3, Table 3), using TFCE and $p<0.01$ uncorrected. The bar graph in Figure 3 shows the distribution across genotype and gender for this region. Not surprisingly, the interaction was significant $(p<0.001)$, but the main effects of genotype and gender were not. No significant interaction between APOE genotype and gender was observed in the anterior DMN. Given the significant interaction in the posterior DMN, post hoc voxelwise testing was performed for this network (using TFCE and $p<0.01$ uncor- rected) for the following three specific contrasts: female $\varepsilon 3$ homozygotes $>$ female $\varepsilon 4$ carriers; male $\varepsilon 3$ homozygotes $>$ male $\varepsilon 4$ carriers; and male $\varepsilon 4$ carriers $>$ female $\varepsilon 4$ carriers. The results showed the most prominent reduction in posterior default mode functional connectivity in female $\varepsilon 4$ carriers compared with female $\varepsilon 3$ homozygotes, mainly in a large (>2000 voxel) cluster encompassing the cuneal cortex, precuneus, and posterior cingulate gyrus. Male $\varepsilon 4$ carriers showed decreased posterior default mode connectivity only in a small (16 voxel) cluster in the left superior parietal cortex compared with male $\varepsilon 3$ homozygotes. In addition, female $\varepsilon 4$ carriers also showed reduced functional connectivity compared with male $\varepsilon 4$ carriers in a cuneus/precuneus cluster of the posterior DMN. See Figure 4 and Table 3 for specifics of the post hoc results.

\section{$A P O E \varepsilon 4$ effect on gray matter volume}

We also tested the effect of $\varepsilon 4$ on gray matter volume. No significant between-group differences or APOE genotype-by-gender interactions were found.
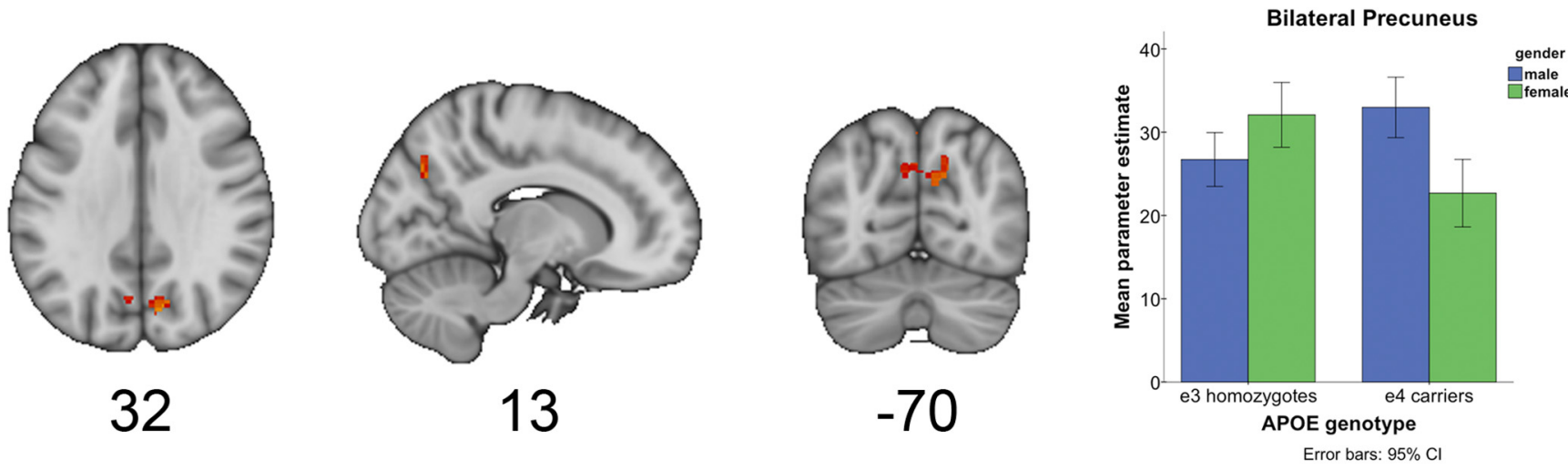

Figure 3. Gender modulates the APOE effect on default mode connectivity. An APOE genotype-by-gender interaction was found in a precuneus/cuneus region within the posterior DMN. The bar graph shows the mean parameter estimates of this cluster, for male and female $\varepsilon 3$ homozygotes and male and female $\varepsilon 4$ carriers. The greatest reduction in functional connectivity was observed in the female $\varepsilon 4$ carriers. The statistical maps, thresholded using TFCE and $p<0.01$ uncorrected, are overlaid on the MNI 152 brain; MNI coordinates (in millimeters) of the slices are displayed. Images are displayed in neurological convention.

Table 3. Brain clusters showing interaction between APOE genotype and gender and clusters showing differences in subsequent post hoc comparisons

\begin{tabular}{|c|c|c|c|c|c|c|}
\hline \multirow[b]{2}{*}{ Brain network } & \multirow[b]{2}{*}{ Contrast } & \multirow[b]{2}{*}{ Brain region } & \multirow[b]{2}{*}{ Cluster size (voxels) } & \multicolumn{3}{|c|}{ Peak MNI coordinates $(\mathrm{mm})$} \\
\hline & & & & $x$ & $y$ & $z$ \\
\hline \multirow[t]{2}{*}{ Posterior DMN } & \multirow[t]{2}{*}{$A P O E \times$ gender interaction } & RH cuneal cortex/precuneus & 73 & 12 & -72 & 32 \\
\hline & & LH precuneus & 49 & 0 & -70 & 38 \\
\hline \multirow[t]{4}{*}{ Posterior DMN } & \multirow[t]{4}{*}{ Female $\epsilon 3$ homozygotes $>$ female $\epsilon 4$ carriers } & RH cuneal cortex/precuneus/posterior cingulate cortex & 2193 & 18 & -74 & 32 \\
\hline & & LH lateral occipital cortex & 52 & -44 & -64 & 16 \\
\hline & & LH supramarginal gyrus & 36 & -50 & -46 & 42 \\
\hline & & LH middle frontal gyrus & 13 & -38 & 12 & 50 \\
\hline Posterior DMN & Male $\epsilon 3$ homozygotes $>$ male $\epsilon 4$ carriers & LH superior parietal lobule & 16 & -28 & -48 & 60 \\
\hline \multirow[t]{3}{*}{ Posterior DMN } & \multirow[t]{3}{*}{ Male $\epsilon 4$ carriers $>$ female $\epsilon 4$ carriers } & RH angular gyrus & 85 & 62 & -50 & 40 \\
\hline & & LH lateral occipital cortex & 30 & -52 & -74 & 40 \\
\hline & & RH cuneal cortex/precuneus & 21 & 12 & -74 & 32 \\
\hline
\end{tabular}


A

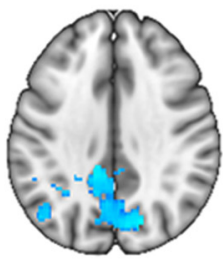

35

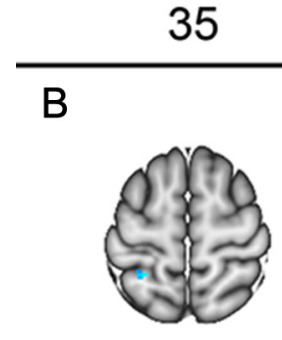

60

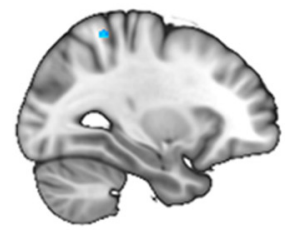

$-29$

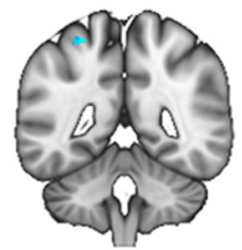

$-45$

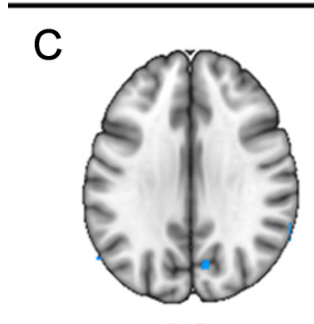

30

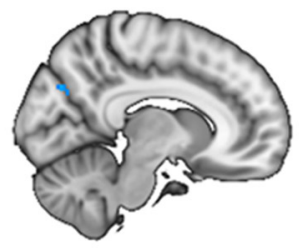

10

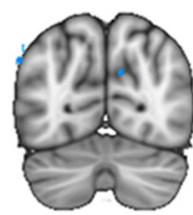

$-70$
Figure 4. The $A P O E$ effect on functional connectivity is most prominent in female $\varepsilon 4$ carriers. $\boldsymbol{A}-\boldsymbol{C}$, Post hoc tests were performed for the posterior DMN for three contrasts: female $\varepsilon 3$ homozygotes $>$ female $\varepsilon 4$ carriers $(\boldsymbol{A})$, which showed a decrease in functional connectivity in female $\varepsilon 4$ carriers, mainly in a large ( $>2000$ voxel) cluster encompassing the cuneal cortex, precuneus, and posterior cingulate gyrus; male $\varepsilon 3$ homozygotes $>$ male $\varepsilon 4$ carriers $(\boldsymbol{B})$, which showed decreased posterior default mode connectivity in male $\varepsilon 4$ carriers in a small (16 voxel) cluster in the left superior parietal cortex; and male $\varepsilon 4$ carriers $>$ female $\varepsilon 4$ carriers $(\boldsymbol{C})$, which showed small clusters of reduced functional connectivity in female $\varepsilon 4$ carriers. The statistical maps, thresholded using TFCE and $p<0.01$ uncorrected, are overlaid on the MNI152 brain; MNI coordinates (in millimeters) of the slices are displayed. Images are displayed in neurological convention.

\section{Discussion}

$A P O E \varepsilon 4$ is a potent $\mathrm{AD}$ risk factor. In the normal population, $10-15 \%$ of persons carry the $\varepsilon 4$ allele; among $\mathrm{AD}$ patients, up to $65 \%$ are $\varepsilon 4$ carriers (Saunders et al., 1993; Farrer et al., 1997). The mechanism through which $\varepsilon 4$ exerts its effect remains elusive, but possibilities include promotion of tau hyperphosphorylation, reduction of $\beta$-amyloid clearance, and inhibition of neurite outgrowth (Kim et al., 2009). Previous studies of AD risk have shown that the $\varepsilon 4$ effect is more pronounced in women (Payami et al., 1996; Farrer et al., 1997; Bretsky et al., 1999). With notable exceptions (Corder et al., 2004; Fleisher et al., 2005; Lehmann et al., 2006), most studies have ignored this gender effect. No previous studies have investigated whether the interaction between gender and $A P O E$ is detectable in healthy adults. Here we have shown that this interaction is detectable in healthy older adults using two distinct $\mathrm{AD}$ biomarkers in two independent samples.

DMN connectivity is a well replicated imaging biomarker of $\mathrm{AD}$ (Greicius et al., 2004; Buckner et al., 2005; Damoiseaux et al., 2011). Our most compelling imaging results demonstrate an APOE genotypeby-gender interaction on posterior DMN connectivity. The precuneus/cuneus region of the posterior DMN showed a significant interaction, with the greatest reduction of functional connectivity in female $\varepsilon 4$ carriers (Fig. 3). Post hoc testing was most notable for a $2000+$ voxel cluster in the posterior cingulate/precuneus showing reduced connectivity in female $\varepsilon 4$ carriers compared with female $\varepsilon 3$ homozygotes (Fig. $4 A$, Table 3 ). The same comparison in men only showed reduced connectivity in $\varepsilon 4$ carriers in a small DMN cluster (Fig. 4B, Table 3). The small size and unusual location (not a core $\mathrm{DMN}$ region) of this cluster led us to interpret this finding cautiously and to strengthen our hypothesis that the $\varepsilon 4$ effect is mainly driven by female carriers. Comparing female and male $\varepsilon 4$ carriers revealed a decrease in female $\varepsilon 4$ carriers in three small but more strategically located DMN clusters (Fig. 4C, Table 3). In structural and functional connectivity analyses, the posterior cingulate/precuneus region is consistently found to be a core node in the DMN (Hagmann et al., 2008; Buckner et al., 2009). This region is structurally connected to the medial temporal lobes (Greicius et al., 2009), the initial site of tau pathology in AD (Braak and Braak, 1991). It shows reduced glucose metabolism early in $\mathrm{AD}$ and in asymptomatic $\varepsilon 4$ carriers (Minoshima et al., 1995; Reiman et al., 1996). Last, this region shows reduced connectivity in $\mathrm{AD}$ patients compared with controls (Greicius et al., 2004) and in MCI patients compared with controls (Sorg et al., 2007). Thus, the interaction of gender and APOE status occurs in a core $\mathrm{DMN}$ node and in the node most tightly linked to $\mathrm{AD}$.

In the anterior DMN, we did not observe an APOE genotypeby-gender interaction. However, in the cluster highlighted to de$\mathrm{p}-\operatorname{tau}(p=0.645)$. 


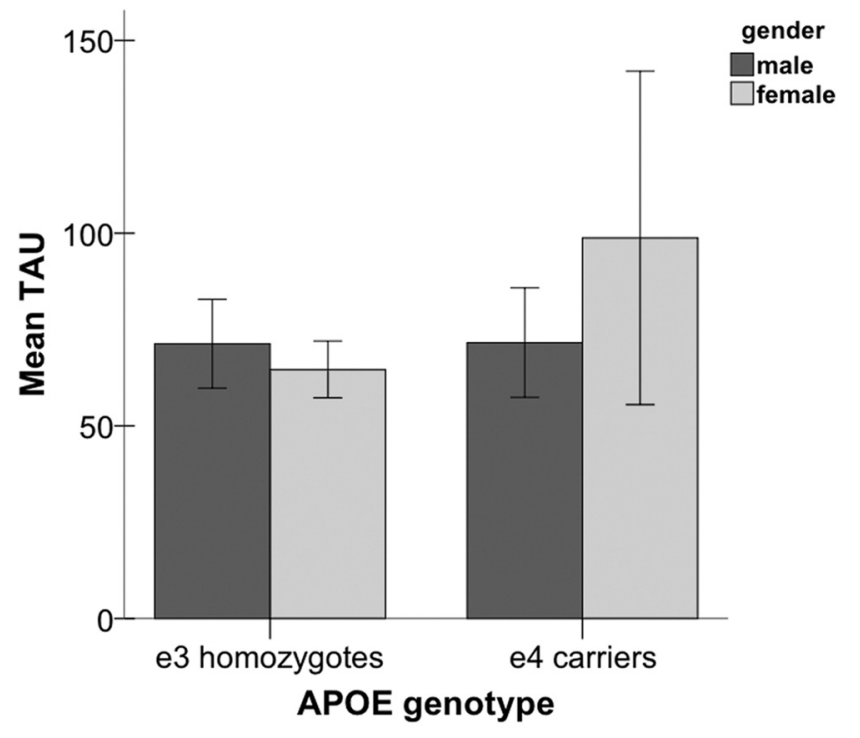

Error bars: $95 \% \mathrm{Cl}$

Figure 5. CSF total tau levels provide convergent evidence for the modulation of the $A P O E$ effect by gender. The bar graph shows the significant $A P O E$ genotype-by-gender interaction observed for CSF total tau levels ( $p=0.024$ ), in the healthy older controls of the ADNI dataset. Healthy female $\varepsilon 4$ carriers showed the highest mean total tau level. Post hoc testing revealed a significant difference between female $\varepsilon 4$ carriers and female $\varepsilon 3$ homozygotes ( $p=0.046$ ).

pict the directionality of the findings (Fig. 2A), we saw additive effects of gender and $A P O E$ genotype. This indicates that, although there is no APOE genotype-by-gender interaction, the additive effects of decreased connectivity in $\varepsilon 4$ carriers and decreased connectivity in women renders female $\varepsilon 4$ carriers most vulnerable to the observed $\varepsilon 4$ effect in this region. The observation of gender differences in resting state functional connectivity, including DMN regions, is consistent with many, but not all (Weissman-Fogel et al., 2010), previous studies on this topic (Liu et al., 2009; Biswal et al., 2010; Allen et al., 2011; Filippi et al., 2012).

Our results may explain the prominent discrepancies in the four previous studies examining the effect of APOE genotype on DMN connectivity in healthy older persons (Sheline et al., 2010; Machulda et al., 2011; Trachtenberg et al., 2011; Westlye et al., 2011). Sheline et al. (2010) found increases and decreases in DMN connectivity in $\varepsilon 4$ carriers, Machulda et al. (2011) only found decreases, Westlye et al. (2011) only found increases, and Trachtenberg et al. (2011) found no differences in the DMN but reported differences in two hippocampal networks. The directionality of our findings is most compatible with that of Machulda et al. (2011), who also showed decreased connectivity in $\varepsilon 4$ carriers. The discrepancy in the findings between our study and the previous four could be due to the different age ranges. The participants in our study and the Machulda et al. (2011) study were older (mean age, $\sim 70$ and 78 years, respectively) than those in the other three studies (mean ages, $\sim 61,63$, and 45 years). Perhaps more important, the ratio of males to females varied considerably across the previous studies. In the Sheline et al. (2010) study, there were 29 female and 9 male $\varepsilon 4$ carriers and 43 female and 19 male $\varepsilon 3$ homozygotes. In the Machulda et al. (2011) study, gender was matched across APOE genotype groups, with 21 females and 35 males in each group. In the Westlye et al. (2011) study, there were 20 female and 13 male $\varepsilon 4$ carriers and 41 female and 21 male $\varepsilon 3$ homozygotes. In the Trachtenberg et al. (2011) study, there were 17 female and 17 male $\varepsilon 4$ carriers and 10 female and 10 male $\varepsilon 3$ homozygotes. The discrepancy in findings across these previous studies might therefore be explained by an underlying and unexplored $A P O E$ genotype-by-gender interaction.

We used the ADNI CSF dataset to obtain convergent evidence of an APOE genotype-by-gender interaction in an independent cohort with an independent biomarker for $\mathrm{AD}$. We found a main effect of $A P O E$ on all three markers in the expected direction and an $A P O E$ genotype-by-gender interaction in total tau levels, in which female $\varepsilon 4$ carriers had significantly elevated levels compared with female $\varepsilon 3$ homozygotes whereas male $\varepsilon 4$ carriers and male $\varepsilon 3$ homozygotes had nearly identical levels. To our knowledge this is the first report of an APOE genotype-by-gender interaction in a CSF biomarker of AD. Numerous studies have shown that AD and MCI patients have reduced CSF levels of $\mathrm{A} \beta_{1-42}$ and increased levels of $\mathrm{p}$-tau and total tau (Tarawneh and Holtzman, 2010). Shaw et al. (2009) have previously examined the ADNI healthy control dataset and noted an APOE effect on $\mathrm{A} \beta_{1-42}$ levels (reduced in $\varepsilon 4$ carriers) and p-tau levels (increased in $\varepsilon 4$ carriers) but did not find a significant $A P O E$ effect in total tau levels (Shaw et al., 2009). Our analysis differs in that we excluded $\varepsilon 2$ carriers and examined an $A P O E$ genotype-by-gender interaction. It is unclear why the $A P O E$ genotype-by-gender interaction was detectable in total tau levels but not in p-tau or $\mathrm{A} \beta_{1-42}$ levels, though this might reflect the temporal progression of biomarkers in AD pathology. It is generally accepted that $\beta$-amyloid pathology precedes tau pathology by many years (Jack et al., 2010, 2011). It is possible that p-tau changes precede total tau changes on the pathological progression to $\mathrm{AD}$. This is consistent with the findings by Shaw et al. (2009), who found that healthy $\varepsilon 4$ carriers, collapsed across gender, differed from non- $\varepsilon 4$ carriers in $\mathrm{A} \beta_{1-42}$ levels and p-tau levels but not (yet) in total tau levels. In our analysis, female $\varepsilon 4$ carriers, presumably further along in their $\mathrm{AD}$ pathology, showed changes in $\mathrm{A} \beta_{1-42}$ levels, p-tau levels, and total tau levels, whereas male $\varepsilon 4$ carriers only manifested differences in the first two markers.

No differences or $A P O E$ genotype-by-gender interactions were observed for gray matter volume or memory performance. Changes in DMN functional connectivity and CSF tau protein levels seem to precede changes in brain structure and cognitive performance. This discrepancy suggests that resting state functional connectivity and CSF protein analyses may therefore be more sensitive than cognitive testing or structural MRI measures as markers of preclinical AD.

Although we show that female $\varepsilon 4$ carriers have decreased DMN connectivity and increased CSF tau levels, we can only speculate about the mechanisms behind this interaction. The most obvious possibility would be some interaction between $A P O E$ status and sex hormone levels. Hundreds of studies in animals and humans have examined the effect of estrogen loss and replacement on cognition and dementia risk in older women (for review, see Henderson, 2009). The negative outcome of the Women's Health Initiative Memory Study, which showed an increased dementia risk in women taking estrogen replacement therapy, has only added to the uncertainty regarding estrogen replacement (Shumaker et al., 2004). Regarding our findings, it is notable that the few studies of estrogen and cognition that have stratified by $A P O E$ status suggest that $A P O E$ status may be critical in evaluating estrogen replacement (Yaffe et al., 2000; Kang and Grodstein, 2010).

We have demonstrated that gender influences the effect of APOE genotype on both DMN connectivity and CSF tau levels and have determined that the detrimental effect of the $\varepsilon 4$ allele is 
most pronounced in women. Our results support previous work showing a higher prevalence of the $\varepsilon 4$ allele among women with $\mathrm{AD}$ and, more critically, demonstrate that this interaction between $A P O E$ genotype and gender is detectable in the preclinical period. Future studies using biomarkers in the preclinical stages of disease should allow us to dissect the environmental, hormonal, and genetic factors that underlie this interaction, which appears central to $\mathrm{AD}$ pathogenesis.

\section{References}

Allen EA, Erhardt EB, Damaraju E, Gruner W, Segall JM, Silva RF, Havlicek M, Rachakonda S, Fries J, Kalyanam R, Michael AM, Caprihan A, Turner JA, Eichele T, Adelsheim S, Bryan AD, Bustillo J, Clark VP, Feldstein Ewing SW, Filbey F, et al. (2011) A baseline for the multivariate comparison of resting state networks. Front Syst Neurosci 5:2.

Andrews-Hanna JR, Reidler JS, Sepulcre J, Poulin R, Buckner RL (2010) Functional-anatomic fractionation of the brain's default network. Neuron 65:550-562.

Ashburner J, Friston KJ (2000) Voxel-based morphometry-the methods. Neuroimage 11:805-821.

Bai F, Watson DR, Shi Y, Wang Y, Yue C, YuhuanTeng, Wu D, Yuan Y, Zhang Z (2011) Specifically progressive deficits of brain functional marker in amnestic type mild cognitive impairment. PLoS One 6:e24271.

Biswal BB, Mennes M, Zuo XN, Gohel S, Kelly C, Smith SM, Beckmann CF, Adelstein JS, Buckner RL, Colcombe S, Dogonowski AM, Ernst M, Fair D, Hampson M, Hoptman MJ, Hyde JS, Kiviniemi VJ, Kötter R, Li SJ, Lin CP, et al. (2010) Toward discovery science of human brain function. Proc Natl Acad Sci U S A 107:4734-4739.

Braak H, Braak E (1991) Neuropathological staging of Alzheimer-related changes. Acta Neuropathol 82:239-259.

Bretsky PM, Buckwalter JG, Seeman TE, Miller CA, Poirier J, Schellenberg GD, Finch CE, Henderson VW (1999) Evidence for an interaction between apolipoprotein E genotype, gender, and Alzheimer disease. Alzheimer Dis Assoc Disord 13:216-221.

Buckner RL, Snyder AZ, Shannon BJ, LaRossa G, Sachs R, Fotenos AF, Sheline YI, Klunk WE, Mathis CA, Morris JC, Mintun MA (2005) Molecular, structural, and functional characterization of Alzheimer's disease: evidence for a relationship between default activity, amyloid, and memory. J Neurosci 25:7709-7717.

Buckner RL, Sepulcre J, Talukdar T, Krienen FM, Liu H, Hedden T, AndrewsHanna JR, Sperling RA, Johnson KA (2009) Cortical hubs revealed by intrinsic functional connectivity: mapping, assessment of stability, and relation to Alzheimer's disease. J Neurosci 29:1860-1873.

Corder EH, Ghebremedhin E, Taylor MG, Thal DR, Ohm TG, Braak H (2004) The biphasic relationship between regional brain senile plaque and neurofibrillary tangle distributions: modification by age, sex, and APOE polymorphism. Ann N Y Acad Sci 1019:24-28.

Damoiseaux JS, Prater KE, Miller BL, Greicius MD (2011) Functional connectivity tracks clinical deterioration in Alzheimer's disease. Neurobiol Aging 33:828.e19-e30.

Farrer LA, Cupples LA, Haines JL, Hyman B, Kukull WA, Mayeux R, Myers RH, Pericak-Vance MA, Risch N, van Duijn CM (1997) Effects of age, sex, and ethnicity on the association between apolipoprotein E genotype and Alzheimer disease: a meta-analysis. APOE and Alzheimer Disease Meta Analysis Consortium. JAMA 278:1349-1356.

Filippi M, Valsasina P, Misci P, Falini A, Comi G, Rocca MA (2012) The organization of intrinsic brain activity differs between genders: a resting state fMRI study in a large cohort of young healthy subjects. Hum Brain Mapp. Advance online publication. Retrieved February 22, 2012. doi:10.1002/hbm.21514.

Filippini N, MacIntosh BJ, Hough MG, Goodwin GM, Frisoni GB, Smith SM, Matthews PM, Beckmann CF, Mackay CE (2009) Distinct patterns of brain activity in young carriers of the APOE-epsilon4 allele. Proc Natl Acad Sci U S A 106:7209-7214.

Fleisher A, Grundman M, Jack CR Jr, Petersen RC, Taylor C, Kim HT, Schiller DH, Bagwell V, Sencakova D, Weiner MF, DeCarli C, DeKosky ST, van Dyck CH, Thal LJ, Alzheimer's Disease Cooperative Study (2005) Sex, apolipoprotein E epsilon 4 status, and hippocampal volume in mild cognitive impairment. Arch Neurol 62:953-957.

Good CD, Johnsrude IS, Ashburner J, Henson RN, Friston KJ, Frackowiak RS
(2001) A voxel-based morphometric study of ageing in 465 normal adult human brains. Neuroimage 14:21-36.

Greicius MD, Srivastava G, Reiss AL, Menon V (2004) Default-mode network activity distinguishes Alzheimer's disease from healthy aging: evidence from functional MRI. Proc Natl Acad Sci U S A 101:4637-4642.

Greicius MD, Supekar K, Menon V, Dougherty RF (2009) Resting state functional connectivity reflects structural connectivity in the default mode network. Cereb Cortex 19:72-78.

Hagmann P, Cammoun L, Gigandet X, Meuli R, Honey CJ, Wedeen VJ, Sporns O (2008) Mapping the structural core of human cerebral cortex. PLoS Biol 6:e159.

Henderson VW (2009) Aging, estrogens, and episodic memory in women. Cogn Behav Neurol 22:205-214.

Jack CR Jr, Knopman DS, Jagust WJ, Shaw LM, Aisen PS, Weiner MW, Petersen RC, Trojanowski JQ (2010) Hypothetical model of dynamic biomarkers of the Alzheimer's pathological cascade. Lancet Neurol 9:119-128.

Jack CR Jr, Vemuri P, Wiste HJ, Weigand SD, Aisen PS, Trojanowski JQ, Shaw LM, Bernstein MA, Petersen RC, Weiner MW, Knopman DS, Alzheimer's Disease Neuroimaging Initiative (2011) Evidence for ordering of Alzheimer disease biomarkers. Arch Neurol 68:1526-1535.

Jenkinson M, Smith S (2001) A global optimisation method for robust affine registration of brain images. Med Image Anal 5:143-156.

Jenkinson M, Bannister P, Brady M, Smith S (2002) Improved optimization for the robust and accurate linear registration and motion correction of brain images. Neuroimage 17:825-841.

Kang JH, Grodstein F (2010) Postmenopausal hormone therapy, timing of initiation, APOE and cognitive decline. Neurobiol Aging. Advance online publication. Retrieved May 8, 2012. doi: org/10.1016/j.neurobiolaging.2010.10.007.

Kim J, Basak JM, Holtzman DM (2009) The role of apolipoprotein E in Alzheimer's disease. Neuron 63:287-303.

Lehmann DJ, Refsum H, Nurk E, Warden DR, Tell GS, Vollset SE, Engedal K, Nygaard HA, Smith AD (2006) Apolipoprotein E epsilon4 and impaired episodic memory in community-dwelling elderly people: a marked sex difference. The Hordaland Health Study. J Neurol Neurosurg Psychiatry 77:902-908.

Littow H, Elseoud AA, Haapea M, Isohanni M, Moilanen I, Mankinen K, Nikkinen J, Rahko J, Rantala H, Remes J, Starck T, Tervonen O, Veijola J, Beckmann C, Kiviniemi VJ (2010) Age-related differences in functional nodes of the brain cortex-a high model order group ICA study. Front Syst Neurosci 4:32.

Liu H, Stufflebeam SM, Sepulcre J, Hedden T, Buckner RL (2009) Evidence from intrinsic activity that asymmetry of the human brain is controlled by multiple factors. Proc Natl Acad Sci U S A 106:20499-20503.

Machulda MM, Jones DT, Vemuri P, McDade E, Avula R, Przybelski S, Boeve BF, Knopman DS, Petersen RC, Jack CR Jr (2011) Effect of APOE $\varepsilon 4$ status on intrinsic network connectivity in cognitively normal elderly subjects. Arch Neurol 68:1131-1136.

Minoshima S, Frey KA, Koeppe RA, Foster NL, Kuhl DE (1995) A diagnostic approach in Alzheimer's disease using three-dimensional stereotactic surface projections of fluorine-18-FDG PET. J Nucl Med 36:1238-1248.

Nichols TE, Holmes AP (2002) Nonparametric permutation tests for functional neuroimaging: a primer with examples. Hum Brain Mapp 15:1-25.

Oakes TR, Fox AS, Johnstone T, Chung MK, Kalin N, Davidson RJ (2007) Integrating VBM into the general linear model with voxelwise anatomical covariates. Neuroimage 34:500-508.

Payami H, Zareparsi S, Montee KR, Sexton GJ, Kaye JA, Bird TD, Yu CE, Wijsman EM, Heston LL, Litt M, Schellenberg GD (1996) Gender difference in apolipoprotein E-associated risk for familial Alzheimer disease: a possible clue to the higher incidence of Alzheimer disease in women. Am J Hum Genet 58:803-811.

Qin P, Liu Y, Shi J, Wang Y, Duncan N, Gong Q, Weng X, Northoff G (2012) Dissociation between anterior and posterior cortical regions during selfspecificity and familiarity: a combined fMRI-meta-analytic study. Hum Brain Mapp 33:154-164

Reiman EM, Caselli RJ, Yun LS, Chen K, Bandy D, Minoshima S, Thibodeau SN, Osborne D (1996) Preclinical evidence of Alzheimer's disease in persons homozygous for the epsilon 4 allele for apolipoprotein E. N Engl J Med 334:752-758.

Rueckert D, Sonoda LI, Hayes C, Hill DL, Leach MO, Hawkes DJ (1999) 
Nonrigid registration using free-form deformations: application to breast MR images. IEEE Trans Med Imaging 18:712-721.

Saunders AM, Strittmatter WJ, Schmechel D, George-Hyslop PH, PericakVance MA, Joo SH, Rosi BL, Gusella JF, Crapper-MacLachlan DR, Alberts MJ (1993) Association of apolipoprotein E allele epsilon 4 with lateonset familial and sporadic Alzheimer's disease. Neurology 43: 1467-1472.

Scott WK, Saunders AM, Gaskell PC, Locke PA, Growdon JH, Farrer LA, Auerbach SA, Roses AD, Haines JL, Pericak-Vance MA (1997) Apolipoprotein E epsilon2 does not increase risk of early-onset sporadic Alzheimer's disease. Ann Neurol 42:376-378.

Shaw LM, Vanderstichele H, Knapik-Czajka M, Clark CM, Aisen PS, Petersen RC, Blennow K, Soares H, Simon A, Lewczuk P, Dean R, Siemers E, Potter W, Lee VM, Trojanowski JQ, Alzheimer's Disease Neuroimaging Initiative (2009) Cerebrospinal fluid biomarker signature in Alzheimer's disease neuroimaging initiative subjects. Ann Neurol 65:403-413.

Sheline YI, Morris JC, Snyder AZ, Price JL, Yan Z, D’Angelo G, Liu C, Dixit S, Benzinger T, Fagan A, Goate A, Mintun MA (2010) APOE4 allele disrupts resting state fMRI connectivity in the absence of amyloid plaques or decreased CSF Abeta42. J Neurosci 30:17035-17040.

Shumaker SA, Legault C, Kuller L, Rapp SR, Thal L, Lane DS, Fillit H, Stefanick ML, Hendrix SL, Lewis CE, Masaki K, Coker LH, Women's Health Initiative Memory Study (2004) Conjugated equine estrogens and incidence of probable dementia and mild cognitive impairment in postmenopausal women: Women's Health Initiative Memory Study. JAMA 291:2947-2958

Smith SM (2002) Fast robust automated brain extraction. Hum Brain Mapp 17:143-155.

Smith SM, Nichols TE (2009) Threshold-free cluster enhancement: addressing problems of smoothing, threshold dependence and localisation in cluster inference. Neuroimage 44:83-98.

Smith SM, Jenkinson M, Woolrich MW, Beckmann CF, Behrens TE, Johansen-Berg H, Bannister PR, De Luca M, Drobnjak I, Flitney DE, Niazy RK, Saunders J, Vickers J, Zhang Y, De Stefano N, Brady JM, Matthews PM (2004) Advances in functional and structural MR image analysis and implementation as FSL. Neuroimage 23 [Suppl 1]:S208-S219.

Sorg C, Riedl V, Mühlau M, Calhoun VD, Eichele T, Läer L, Drzezga A, Förstl H, Kurz A, Zimmer C, Wohlschläger AM (2007) Selective changes of resting state networks in individuals at risk for Alzheimer's disease. Proc Natl Acad Sci U S A 104:18760-18765.
Strittmatter WJ, Saunders AM, Schmechel D, Pericak-Vance M, Enghild J, Salvesen GS, Roses AD (1993) Apolipoprotein E: high-avidity binding to beta-amyloid and increased frequency of type 4 allele in late-onset familial Alzheimer disease. Proc Natl Acad Sci U S A 90:1977-1981.

Tarawneh R, Holtzman DM (2010) Biomarkers in translational research of Alzheimer's disease. Neuropharmacology 59:310-322.

Trachtenberg AJ, Filippini N, Ebmeier KP, Smith SM, Karpe F, Mackay CE (2012) The effects of APOE on the functional architecture of the resting brain. Neuroimage 59:565-572.

Uddin LQ, Kelly AM, Biswal BB, Xavier Castellanos F, Milham MP (2009) Functional connectivity of default mode network components: correlation, anticorrelation, and causality. Hum Brain Mapp 30:625-637.

Veer IM, Beckmann CF, van Tol MJ, Ferrarini L, Milles J, Veltman DJ, Aleman A, van Buchem MA, van der Wee NJ, Rombouts SA (2010) Whole brain resting state analysis reveals decreased functional connectivity in major depression. Front Syst Neurosci 4:41.

Wang K, Liang M, Wang L, Tian L, Zhang X, Li K, Jiang T (2007) Altered functional connectivity in early Alzheimer's disease: a resting state fMRI study. Hum Brain Mapp 28:967-978.

Weissman-Fogel I, Moayedi M, Taylor KS, Pope G, Davis KD (2010) Cognitive and default-mode resting state networks: do male and female brains "rest" differently? Hum Brain Mapp 31:1713-1726.

Westlye ET, Lundervold A, Rootwelt H, Lundervold AJ, Westlye LT (2011) Increased hippocampal default mode synchronization during rest in middle-aged and elderly APOE epsilon4 carriers: relationships with memory performance. J Neurosci 31:7775-7783.

Whitfield-Gabrieli S, Moran JM, Nieto-Castañón A, Triantafyllou C, Saxe R, Gabrieli JD (2011) Associations and dissociations between default and self-reference networks in the human brain. Neuroimage 55:225-232.

Yaffe K, Haan M, Byers A, Tangen C, Kuller L (2000) Estrogen use, APOE, and cognitive decline: evidence of gene-environment interaction. Neurology 54:1949-1954.

Zhang Y, Brady M, Smith S (2001) Segmentation of brain MR images through a hidden Markov random field model and the expectationmaximization algorithm. IEEE Trans Med Imaging 20:45-57.

Zhang HY, Wang SJ, Xing J, Liu B, Ma ZL, Yang M, Zhang ZJ, Teng GJ (2009) Detection of PCC functional connectivity characteristics in resting state fMRI in mild Alzheimer's disease. Behav Brain Res 197:103-108. 